\title{
Cisplatin in combination with zoledronic acid: A synergistic effect in triple-negative breast cancer cell lines
}

\author{
TONI IBRAHIM $^{1 *}$ CHIARA LIVERANI $^{1 *}$, LAURA MERCATALI $^{1}$, EMANUELE SACANNA ${ }^{1}$, \\ MICHELE ZANONI ${ }^{1}$, FRANCESCO FABBRI ${ }^{2}$, WAINER ZOLI ${ }^{2}$ and DINO AMADORI ${ }^{1}$ \\ ${ }^{1}$ Osteoncology Center; ${ }^{2}$ Biosciences Laboratory, IRCCS Istituto Scientifico Romagnolo \\ per lo Studio e la Cura dei Tumori (I.R.S.T.), I-47014 Meldola (FC), Italy
}

Received October 9, 2012; Accepted November 20, 2012

DOI: 10.3892/ijo.2013.1809

\begin{abstract}
Zoledronic acid (ZA) is the most widely used bisphosphonate to treat cancer-induced bone disease. There is evidence that bisphosphonates have direct antitumor activity and that their combination with anticancer agents can significantly enhance the effect of treatment. We evaluated whether the combination of ZA with different platinum compounds exerts a synergistic effect in breast cancer cell lines and we investigated the mechanisms of action involved. This study was performed on four breast cancer cell lines, MCF-7, SKBR3, MDA-MB-231 and BRC-230, and confirmed on a primary culture obtained from a breast cancer bone metastasis specimen. ZA $(50 \mu \mathrm{M})$ was administered for $72 \mathrm{~h}$ alone or in combination with cisplatin (Cis) or carboplatin. Drug-induced growth inhibition was detected by sulforhodamine B assay, apoptosis and cell cycle regulation were detected by flow cytometry, and protein expression was evaluated by western blot analysis. MCF-7 and SKBR3 showed very low sensitivity to the three drugs tested. The $\mathrm{ZA}+\mathrm{Cis}$ combination exerted a high antitumor activity in the two triple-negative lines MDA-MB-231 and BRC-230. An important synergistic effect was obtained in MDA-MB-231 and an additive effect was observed in BRC-230. The p21, pMAPK and $\mathrm{m}$-TOR pathways were regulated by this combined treatment, particularly at lower Cis doses. Carboplatin did not show antitumor activity either alone or in combination with ZA. In conclusion, the potential novel treatment schedule identified for triple-negative breast cancer could prove beneficial in view of the limited therapeutic options available for patients and also since the synergism with ZA would enable lower Cis doses to be used, thus reducing toxicity. Although further research in a
\end{abstract}

Correspondence to: Dr Toni Ibrahim, Osteoncology Center, IRCCS Istituto Scientifico Romagnolo per lo Studio e la Cura dei Tumori (I.R.S.T.), Via P. Maroncelli 40, I-47014 Meldola (FC), Italy

E-mail: t.ibrahim@irst.emr.it

${ }^{*}$ Contributed equally

Key words: bone metastasis, breast cancer, platinum compound, preclinical model, m-TOR, zoledronic acid clinical setting is warranted, our results on cell lines has been confirmed on a human primary bone metastasis culture.

\section{Introduction}

Breast cancer is the most frequently diagnosed cancer and the leading cause of cancer-related mortality among women, accounting for $23 \%$ of all new cancer cases and $14 \%$ of cancer deaths (1). Bone is one of the most preferential target sites of metastasis for breast cancer and up to $70 \%$ of women with advanced disease develop bone metastases (2). Such lesions have devastating effects, including pain, pathologic fractures, spinal compression and hypercalcemia, all of which greatly compromise the quality of life and outcome (3).

Since results from large randomized controlled trials were published in the late 1990s, bisphosphonates have become the standard of care for the prevention and treatment of skeletal complications associated with bone metastases in breast cancer (4). The third generation nitrogen-containing bisphosphonate, zoledronic acid (ZA), is the only bisphosphonate licensed for the treatment of bone disease originating from a variety of solid tumors and multiple myeloma (5). ZA reduces osteoclastic bone resorption by inhibiting key enzymes of the mevalonate pathway (6), including farnesyl pyrophosphate synthase (7) and geranylgeranyl pyrophosphate synthase (8), leading to incomplete post translational prenylation of signaling GTPases, including Ras, Rho and Rac (9), which ultimately causes osteoclasts to undergo apoptosis (10).

In addition to their inhibitory effect on osteoclasts, there is increasing preclinical evidence to suggest that bisphosphonates exert a direct antitumor activity comprising inhibition of tumor cell growth, induction of cancer cell apoptosis (11-15), inhibition of tumor cell adhesion and invasion (16-18) and antiangiogenic activity (19). Furthermore, bisphosphonates used in combination with anticancer agents appear to significantly enhance the effect of treatment. In fact, ZA has been shown to synergistically increase breast cancer cell death when combined with doxorubicin, paclitaxel, or cyclophosphamide (20-22).

Several dosing schedules of ZA for the treatment of bone metastases have been proposed; a recent study suggested that metronomic weekly low-dose of ZA could be more effective than the conventional ZA given every 4 weeks (23). We 
previously observed that the anti-proliferative activity of ZA in breast cancer cell lines was enhanced using a repeated treatment schedule rather than a continuous one, and that the difference between the two schedules was statistically relevant only in triple-negative breast cancer lines (1). Triple-negative breast cancer (TNBC), which accounts for approximately $15 \%$ of all breast malignancies, is used to define tumors that lack estrogen and progesterone receptor expression and HER-2 amplification. It is often an aggressive disease characterized by frequent and early relapse, a propensity for visceral involvement and shorter periods of disease-free and overall survival with respect to other breast cancer subgroups. The unfavorable prognosis associated with TNBC and the lack of effective targeted therapy has made it the subject of intensive research in recent years (24). TNBC exhibits an abundance of DNA aberrations, suggesting that DNA repair mechanisms are defective. Consequently, these tumors may have increased sensitivity to agents, such as platins, which cause interstrand DNA breaks. The sensitivity of TNBC to platinum-based chemotherapy has thus been the focus of several recent clinical trials in neoadjuvant, adjuvant and advanced disease settings (25).

The aim of the present study was to investigate the activity of ZA in combination with different platinum compounds in four breast cancer cell lines and to explore the molecular mechanisms of action of the drugs.

\section{Materials and methods}

Cell culture. The experiments were performed on four human breast cancer cell lines. MCF-7, SKBR3 and MDA-MB-231 were obtained from the American Type Culture Collection, (Rockville, MD, USA), while BRC-230 was stabilized and characterized in our laboratory (26). Hormone receptor and HER2 status of the four cell lines are shown in Table I. Cells were cultured as a monolayer in $75-\mathrm{cm}^{2}$ flasks at $37^{\circ} \mathrm{C}$ in $\mathrm{TF}$ medium (45\% HAM F12 and 45\% DMEM) supplemented with $10 \%$ fetal bovine serum, $1 \%$ glutamine and $1 \%$ insulin (Mascia Brunelli S.p.a., Milan, Italy) in a $5 \% \mathrm{CO}_{2}$ atmosphere. Cells were cultured to the exponential growth phase and then treated with ZA alone or in combination with carboplatin or cisplatin (Cis).

Isolation of primary cells from a breast cancer bone metastasis. The tumor material tissue was obtained from a patient undergoing surgery for a bone metastasis of breast carcinoma. The protocol was reviewed and approved by the Local Ethics Committee and performed according to Good Clinical Practice and the Helsinki declaration. The patient provided written informed consent to participate in the study.

The tumor tissue was washed twice in sterile PBS 1X supplemented with $10 \%$ penicillin/streptomycin and $5 \%$ amphotericin. The biopsy was then disaggregated by cutting the sample with sterile surgical blades. The obtained fragments were incubated with collagenase type I (Millipore Corp., Billerica, MA, USA) at $37^{\circ} \mathrm{C}$ in stirring conditions. The enzymatic digestion was stopped after 3-4 $\mathrm{h}$ by adding IMDM medium supplemented with $10 \%$ fetal bovine serum, $1 \%$ glutamine, $10 \%$ penicillin/ streptomycin and 5\% amphotericin and L-glutamine. The samples were allowed to settle on the bottom of the tube to
Table I. Hormone receptor and HER2 status of the four breast cancer cell lines and the primary culture.

\begin{tabular}{lccc}
\hline Cell line & ER & PgR & HER2 \\
\hline BRC-230 & - & - & - \\
MCF-7 & + & + & - \\
MDA-MB 231 & - & - & - \\
SKBR3 & - & - & + \\
Primary tumor & + & + & - \\
\hline
\end{tabular}

separate tissue fragments from collagenase-released cells. The cells were counted and seeded at a density of $10,000 / \mathrm{cm}^{2}$. Hormone receptor and HER2 status of the primary tumor are shown in Table I. Pan cytokeratin immunocytochemistry analysis was performed according to the manufacturer's instructions (Epithelial Detection kit, As-Diagnostik, Hueckeswagen, Germany) to detect the percentage of tumor cells of the sample.

Drugs. Cis (Bristol-Myers Squibb S.p.A, Rome, Italy) was stored at room temperature, carboplatin (Bristol-Myers Squibb S.p.A) at $4^{\circ} \mathrm{C}$, and both drugs were diluted in medium prior to use.ZA (Zometa $\left.{ }^{\circledR}\right)$, kindly provided by Novartis (East Hanover, $\mathrm{NJ}$, USA), was solubilized, stored at $-20^{\circ} \mathrm{C}$ at a concentration of $50 \mathrm{mM}$ in sterile water and diluted in medium prior to use.

Treatment schedules. The four cell lines were exposed to ZA and either platin, singly or in combination, for $72 \mathrm{~h}$. ZA was tested at a concentration of $50 \mu \mathrm{M}$ for $72 \mathrm{~h}$, while Cis and carboplatin were tested at concentrations of $0.001,0.01,0.1,1$ and $10 \mu \mathrm{M}$ and 1,11 and $110 \mu \mathrm{M}$, respectively, for $6 \mathrm{~h}$ followed by a $72-h$ washout. For the combination assays, cell lines were exposed to different concentrations of Cis or carboplatin in combination with ZA $(50 \mu \mathrm{M})$ for $6 \mathrm{~h}$, washed out and then exposed to ZA $(50 \mu \mathrm{M})$ for a further $72 \mathrm{~h}$ (Fig. 1).

Chemosensitivity assay. The sulforhodamine B (SRB) assay was used according to the method by Skehan et al to evaluate the cytotoxic activity of the drugs (27). Briefly, cells were collected by trypsinization, counted and plated at a density of 3,000 cells/well in 96-well flat-bottomed microtiter plates. After $24 \mathrm{~h}$, cells were treated with the different schedules. The optical density (OD) of cells was determined at a wavelength of $540 \mathrm{~nm}$ by a colorimetric plate reader. Growth inhibition and cytocidal effects of the drugs were calculated according to the formula reported by Monks et al (28): (OD treated/OD control) $x 100 \%$ where the OD treated reflects the cell number in treated wells and the OD control reflects the cell number in untreated wells on the day of the assay. If the resulting percentage ratio is above zero, a cytostatic effect has been induced, whereas if it is below zero, cell killing has occurred. The interaction between drugs was evaluated with the method by Kern et al (29), subsequently modified by Romanelli et al (30). The expected survival (defined as the result of the observed survival for drug A alone and the observed survival for drug B alone) and the observed survival for the combination of drugs was used to calculate an $\mathrm{R}$ index $(\mathrm{RI})$ : $\mathrm{S}_{\mathrm{exp}} / \mathrm{S}_{\mathrm{obs}}$. $\mathrm{RI} \leq 0.5$ 


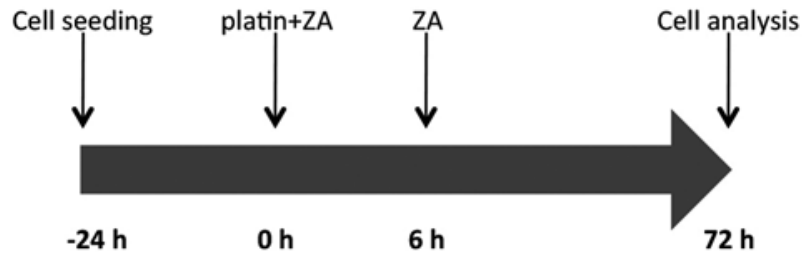

Figure 1. Drug combination schedule.

indicates an antagonistic effect between drugs, whereas $\geq 0.5 \mathrm{RI} \leq 1.5$ indicates an additive effect and $\mathrm{RI} \geq 1.5$ indicates a synergistic effect. Four biological independent replicates of each experiment were performed.

Western blot analysis. Proteins were isolated by cell lysis with a lysis buffer composed of $50 \mathrm{mM}$ Tris- $\mathrm{HCl}(\mathrm{pH} \mathrm{8.0)}$, $150 \mathrm{mM} \mathrm{NaCl}, 1 \%$ Triton X-100 and $0.1 \%$ SDS, supplemented with $1 \mathrm{mM}$ phenylmethylsulfonyl fluoride and 1:100 protease inhibitors (Sigma-Aldrich). The protein content was quantified using the BCA protein assay kit (Thermo Fisher Scientific, Waltman, MA, USA). An equal amount of protein from each sample was separated on Criterion ${ }^{\mathrm{TM}}$ Precast Gel Tris- $\mathrm{HCl}$ (Bio-Rad, Hercules, CA, USA) and transferred to polyvinylidene fluoride membranes (Millipore). The membranes were blocked for $2 \mathrm{~h}$ in $5 \%$ non-fat dry milk PBS with $0.1 \%$ Tween-20 (Sigma-Aldrich, Steinheim, Germany) at room temperature and incubated overnight at $4^{\circ} \mathrm{C}$ with primary antibody. After washing, the membranes were incubated for $1 \mathrm{~h}$ at room temperature with horseradish peroxidase-conjugated secondary antibody. The following primary antibodies were used: anti-RAS (polyclonal, 1:1000) (Stressgen, Brussels, Belgium), anti-p-MAPK (polyclonal, 1:1000), anti pM-TOR (1:1000) (Cell Signaling Technology, Inc., Beverly, MA, USA), anti-caspase-3 (polyclonal, 1:500), anti-caspase-8 (monoclonal, 1:500) (Alexis Biochemicals, Farmingdale, NY, USA), anti-caspase-9 (polyclonal, 1:500), anti-Mcl-1 (monoclonal 1:100) (BD Pharmingen, San Diego, CA, USA), anti-bcl-2 (monoclonal, 1:100) (Dako Corp., Glostrup, Denmark), antip21 (monoclonal, 1:100) (BioOptica, Milan, Italy), anti-Rho (monoclonal 1:1000) (Millipore), and anti-actin (polyclonal, 1:5000) (Sigma-Aldrich).

TUNEL assay. Fragmented DNA generated in response to apoptotic signals was detected by the terminal deoxynucleotidyl transferase (TdT) nick-end labeling (TUNEL) assay. After each treatment schedule, $10^{6}$ cells were washed twice with PBS, fixed by incubation in $1 \%$ formaldehyde on ice for $15 \mathrm{~min}$, resuspended in $70 \%$ ice cold ethanol and stored overnight. Cells were then washed twice in PBS and resuspended in PBS containing $0.1 \%$ Triton $\mathrm{X}-100$ for $5 \mathrm{~min}$ at $48^{\circ} \mathrm{C}$. Thereafter, samples were incubated in $50 \mathrm{ml}$ of solution containing TdT and FITC conjugated dUTP deoxynucleotides 1:1 (Roche Diagnostics GmbH, Mannheim, Germany) in a humidified atmosphere for $90 \mathrm{~min}$ at $37^{\circ} \mathrm{C}$ in the dark, washed in PBS, counterstained with propidium iodide $(2.5 \mathrm{mg} / \mathrm{ml}, \mathrm{MP}$ Biomedicals, Verona, Italy) and RNAse $(10 \mathrm{kU} / \mathrm{ml}$, SigmaAldrich) for $30 \mathrm{~min}$ at $48^{\circ} \mathrm{C}$ in the dark and analyzed by flow cytometry.
Cell cycle analysis. After all the treatment schedules, cells were fixed in ethanol (70\%), stained in a solution containing propidium iodide (10 $\mathrm{mg} / \mathrm{ml}$, MP Biomedicals), RNAse (10 kU/ml, Sigma-Aldrich) and NP40 (0.01\%, Sigma-Aldrich) overnight at $48^{\circ} \mathrm{C}$ in dark conditions and analyzed by flow cytometry. Data were expressed as fractions of cells in the different cell cycle phases.

Scratch wound assay. We used a scratch wound assay to evaluate the migration ability of the four cell lines after treatment. Cells were cultured in $75-\mathrm{cm}^{2}$ flasks, as previously described, and were exposed to the different treatment schedules. Twenty-four hours before the end of treatment, a uniform cell-free area was created by scratching a confluent monolayer with a scraper. The migration rate of the cell lines was determined by observing the wound closure at the end of the experiments (31).

Statistical analysis. Differences between treatments in terms of dose-response, apoptosis and cell cycle block were determined using the Student's t-test for unpaired observations. $\mathrm{P}<0.05$ was considered to indicate statistically significant differences. In each experiment the standard deviation did not exceed $10 \%$.

\section{Results}

Drug sensitivity. Drug combination experiments were performed using one dose of $\mathrm{ZA}(50 \mu \mathrm{M})$ for $72 \mathrm{~h}$ and five doses of Cis $(0.001,0.01,0.1,1$ and $10 \mu \mathrm{M})$ or three doses of carboplatin $(1,11$ and $110 \mu \mathrm{M})$. The hormone receptor-positive line MCF-7 and HER-2 expressing line SKBR3 showed very low sensitivity to all the drugs tested, whether alone or in combination (data not shown). Conversely, the Cis and ZA combination showed a high anti-proliferative effect in the triple-negative cell lines BRC-230 and MDA-MB-231, the latter proving the most sensitive to treatment. $\mathrm{IG}_{50}$ was reached at $<0.001 \mu \mathrm{M}$ with the combination, whereas it was not reached with Cis alone, even at the highest concentration (Fig. 2A). BRC-230 cells were more sensitive than MDA-MB-231 to Cis alone, with an $\mathrm{IG}_{50}$ of $4.6 \mu \mathrm{M}$. However, the increase in growth inhibition obtained with the drug combination was lower than that observed for MDA-MB-231, with an $\mathrm{IG}_{50}$ of $0.005 \mu \mathrm{M}$ (Fig. 2B). Both triplenegative lines proved insensitive to carboplatin treatment, alone or in combination with ZA (data not shown). No synergistic or additive effects were observed when carboplatin or Cis were combined with ZA in MCF-7 or SKBR3. In MDA-MB-231, the combination of ZA and Cis produced an important synergistic effect which yielded an R index $>1.5$ for all but the $10-\mu \mathrm{M}$ Cis concentration. The synergism was particularly evident at lower concentrations of the platin (0.001 and $0.01 \mu \mathrm{M})$ (Fig. 2C). An additive effect was reached when combining $\mathrm{Cis}$ and ZA in BRC-230 for all Cis concentrations, and the interaction was once again higher at lower concentrations of the drug (Fig. D). Conversely, the combination of carboplatin and ZA did not produce either additive or synergistic effects and the increase in growth inhibition obtained with the drug combination was similar to that obtained with ZA alone (data not shown). Based on these results, we performed subsequent experiments using the $\mathrm{Cis}$ and $\mathrm{ZA}$ combination in the triple-negative cell lines BRC-230 and MDA-MB-231. 
A

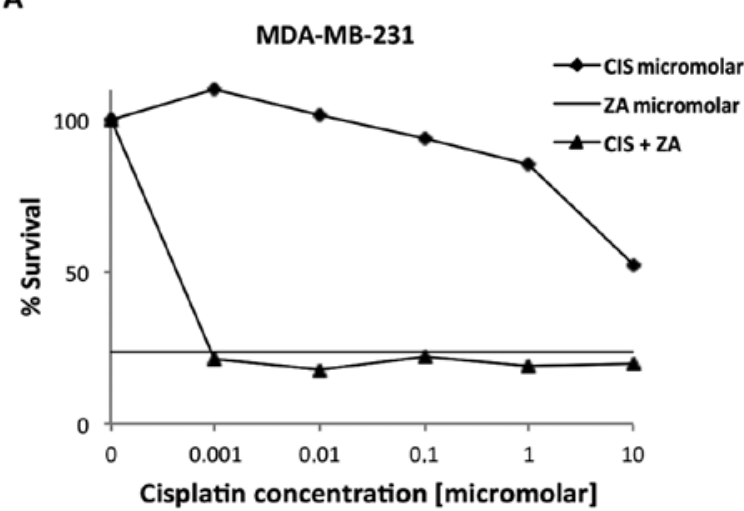

C

MDA-MB-231

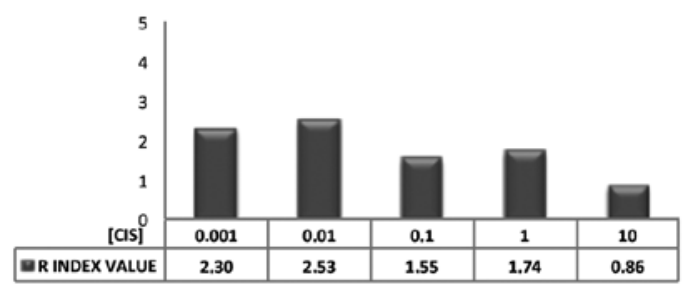

B

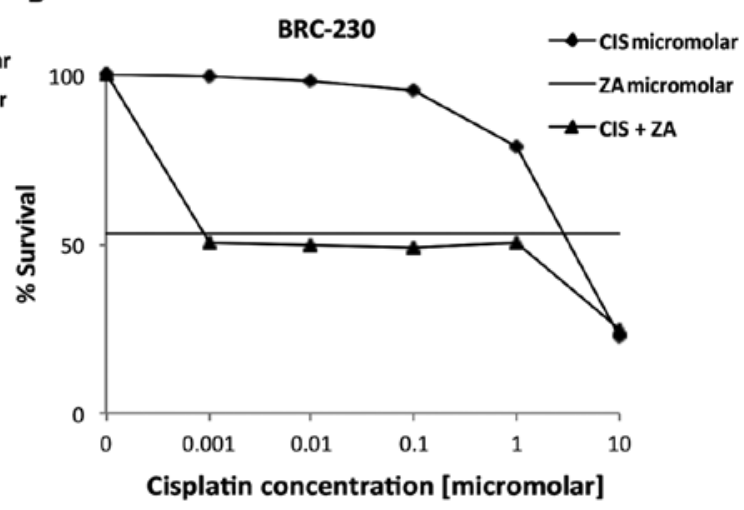

D

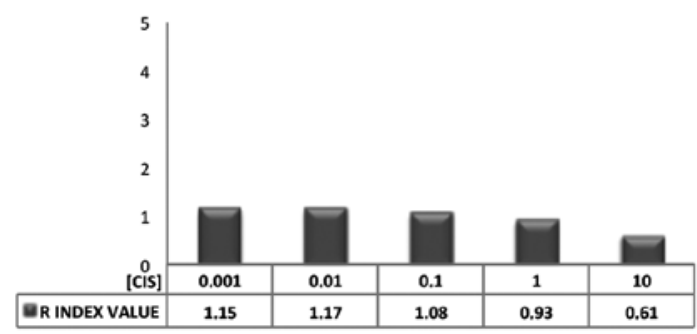

Figure 2. Dose-response curves of MDA-MB-231 (A) and BRC-230 (B) treated with different concentrations of Cis and/or ZA $50 \mu \mathrm{M}$ for $72 \mathrm{~h}$. Data represent the means of three independent experiments. R-index values obtained with the combination of ZA (50 $\mu \mathrm{M})$ and different Cis concentrations in MDA-MB-231 (C) and BRC-230 (D). Data represent the means of three independent experiments.

Isolation of primary cancer cells from breast bone metastasis biopsy. The primary culture obtained from the surgical material was stable for 4-5 subcultures. In order to verify the presence of tumor cells in the surgical material, the cytospin sections were stained for pan cytokeratin immunocytochemistry assay. The percentage of cells expressing an epithelial phenotype was $20 \%$ of the whole culture (Fig. 3).

Drug sensitivity of primary culture. The drug sensitivity data of Cis and ZA were compared with the values obtained for the cells isolated from the bone metastasis biopsy. The $\mathrm{IG}_{50}$ obtained for the primary culture was similar to the one obtained for MCF-7, the cell line that presents the same HER-2 and hormone receptor pattern. The primary culture proved to be more sensitive to Cis alone with respect to $\mathrm{MCF}-7, \mathrm{IG}_{50}$ of $8.0 \mu \mathrm{M}$ for the primary culture whereas not reached for MCF-7. However, the two cultures showed similar sensitivity for ZA, $\mathrm{IG}_{50}$ not reached for both cell lines, and for the combination of Cis and $\mathrm{ZA}$ with an $\mathrm{IG}_{50}$ of $6.6 \mu \mathrm{M}$ for $\mathrm{MCF}-7$ and $6.9 \mu \mathrm{M}$ for the primary culture.

Effect on proliferation pathways. A strong reduction in pMAPK levels was observed in BRC-230 after the Cis and ZA combination with respect to control cells, especially at the lowest Cis dose $(0.001 \mu \mathrm{m})$. Such a reduction did not occur in single treatments. Furthermore, MCL-1 expression was downregulated in the MDA-MB-231 cell line after the combined treatment but not after single drug exposure. Finally, pM-TOR was markedly downregulated in the MDA-MB-231 cell line following exposure to ZA alone and especially after combined treatment with any of the Cis concentrations (Fig. 4).
Apoptosis induction. Assessment of apoptosis by TUNEL assay showed that both single drug exposure and the ZA and Cis combination induced a small, not statistically significant increase in apoptotic cell percentage with respect to control in both cell lines. In MDA-MB-231, the apoptotic cell percentage did not exceed $5 \%$ in any of the Cis concentrations used alone or in combination with ZA (Fig. 5A). In BRC-230 the percentage of apoptosis reached 7.7 and $6.3 \%$ after the combination of $\mathrm{ZA}$ and $\mathrm{Cis} 0.01$ or $0.1 \mu \mathrm{M}$, respectively (Fig. 5B). These data are in agreement with western blot analysis of caspase-3, -8 and -9 . We did not observe a substantial increase in the cleaved form of the three caspases or a decrease in pro-caspase levels after any of the treatments (Fig. 5C).

Cell cycle perturbation. The combination of ZA and Cis did not produce a significant block of the cell cycle in the G0-G1 or G2 phases in either triple-negative cell line. A slight increment with respect to control was observed in the percentage of cells in G0-G1 after treatment with ZA alone and also in combination with all Cis concentrations in MDA-MB-231 (Fig. 6A) and with Cis 0.001 and $0.01 \mu \mathrm{m}$ in BRC-230 (Fig. 6B). These findings were confirmed by western blot analysis of $\mathrm{p}-21$ in which the protein was found to be upregulated with respect to control after treatment with ZA and Cis at any tested dose in the MDA-MB-231 cell line, but only at the lowest $\mathrm{Cis}$ doses for BRC-230 (Fig. 6C).

Effect on migration ability. Treatment of cells with the combination of $\mathrm{ZA}$ and $\mathrm{Cis}$ resulted in a decreased migration rate with respect to control cells. Such a reduction was detected using the scratch assay in both triple-negative cell lines. Untreated cells 
A

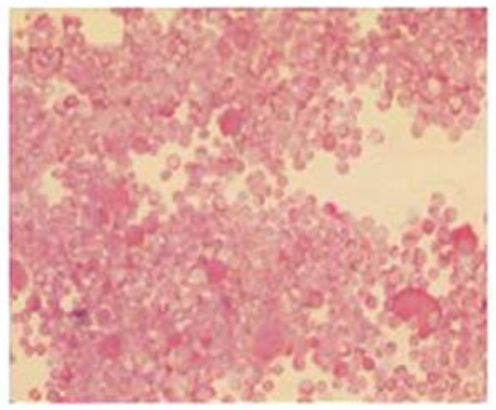

B

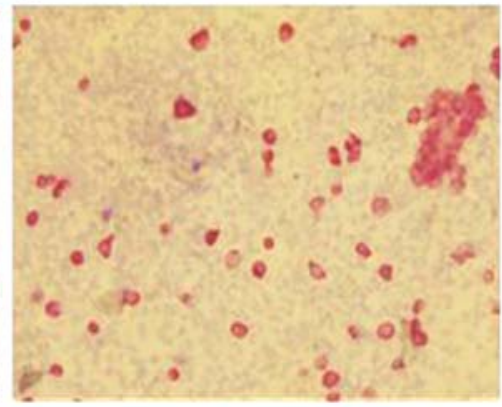

Figure 3. MCF-7 (A) and primary culture of bone metastasis lesion cells (B) positive stained for Pan cytokeratin in immunocytochemistry assay.

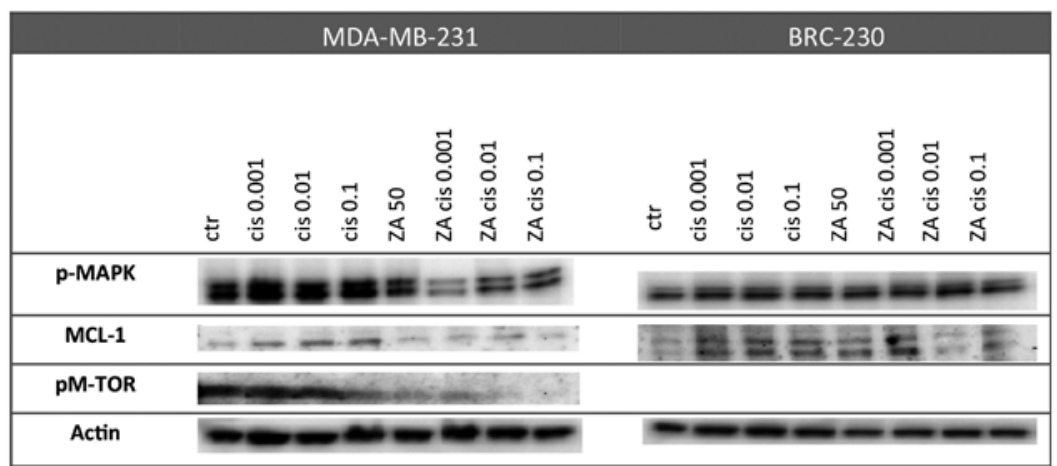

Concentration in micromolar

Figure 4. Protein expression levels of p-MAPK and MCL-1 detected by western blot analysis before and after treatment with Cis $(0.001,0.01$ and $0.1 \mu \mathrm{M})$ and/ or ZA $50 \mu \mathrm{M}$.

A

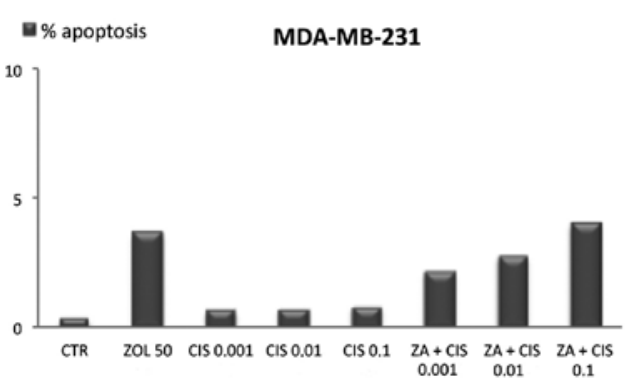

B

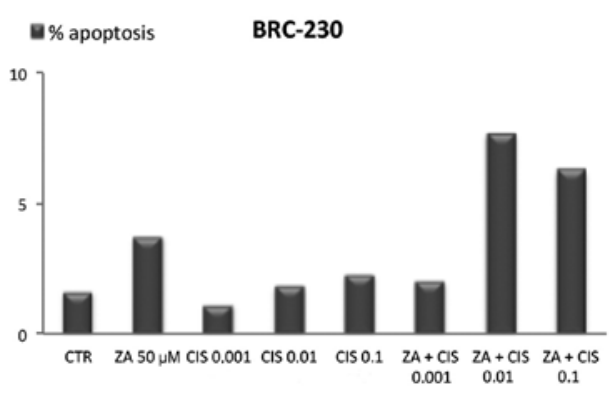

C

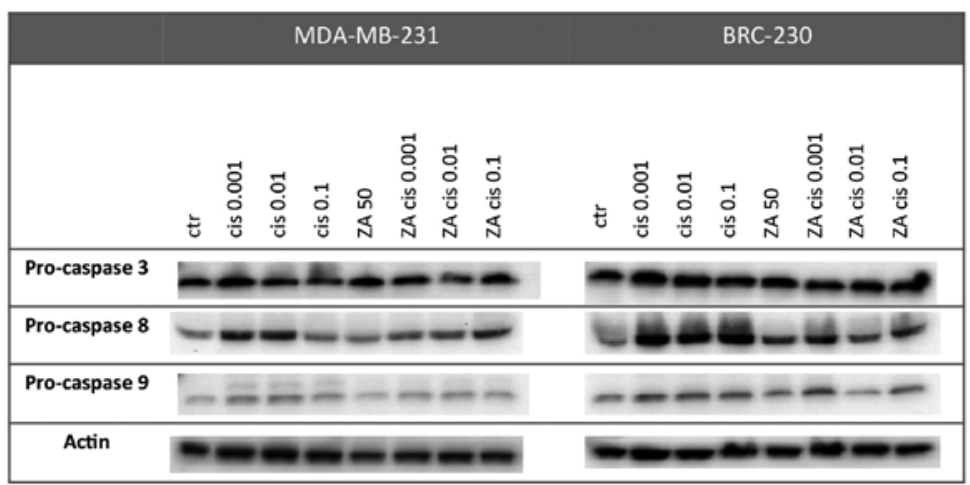

Concentration in micromolar

Figure 5. Apoptotic cells detected by TUNEL assay before and after single or combined treatments in MDA-MB-231 (A) and BRC-230 (B). Data represent the means of three independent experiments. Protein expression levels of pro-caspase-3, -8 and -9 detected by western blot analysis before and after single or combined treatments $(\mathrm{C})$. 
A

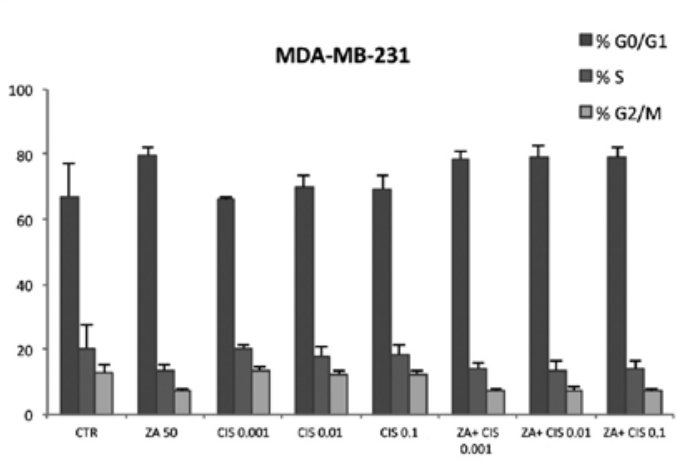

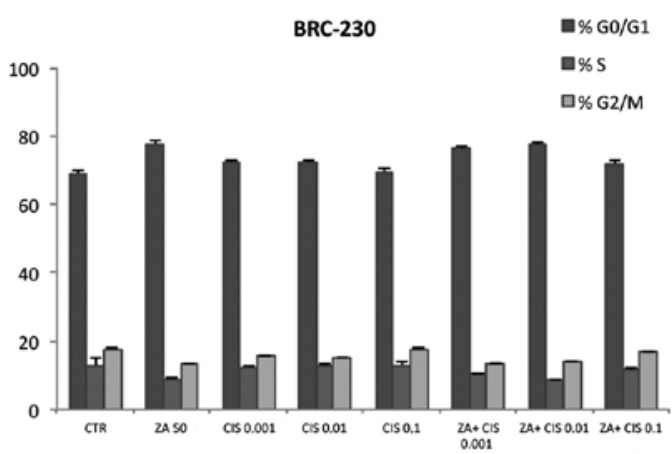

C

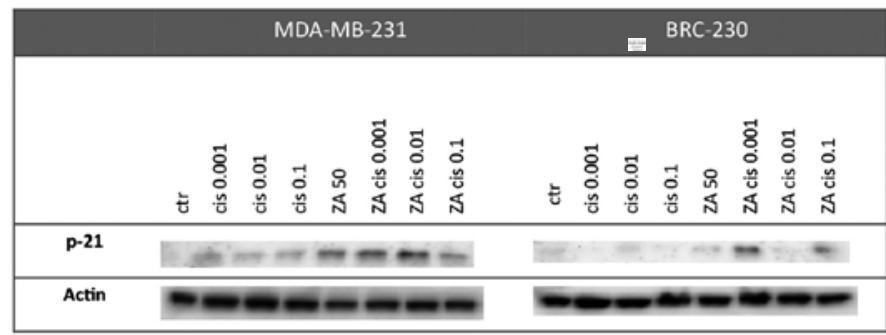

Concentration in micromolar

Figure 6. Cell distribution in different cell cycle phases before and after single or combined treatments in MDA-MB-231 (A) and in BRC-230 (B). Data represent the means of three independent experiments. Protein expression levels of p-21 detected by western blot analysis before and after single or combined treatments $(\mathrm{C})$.

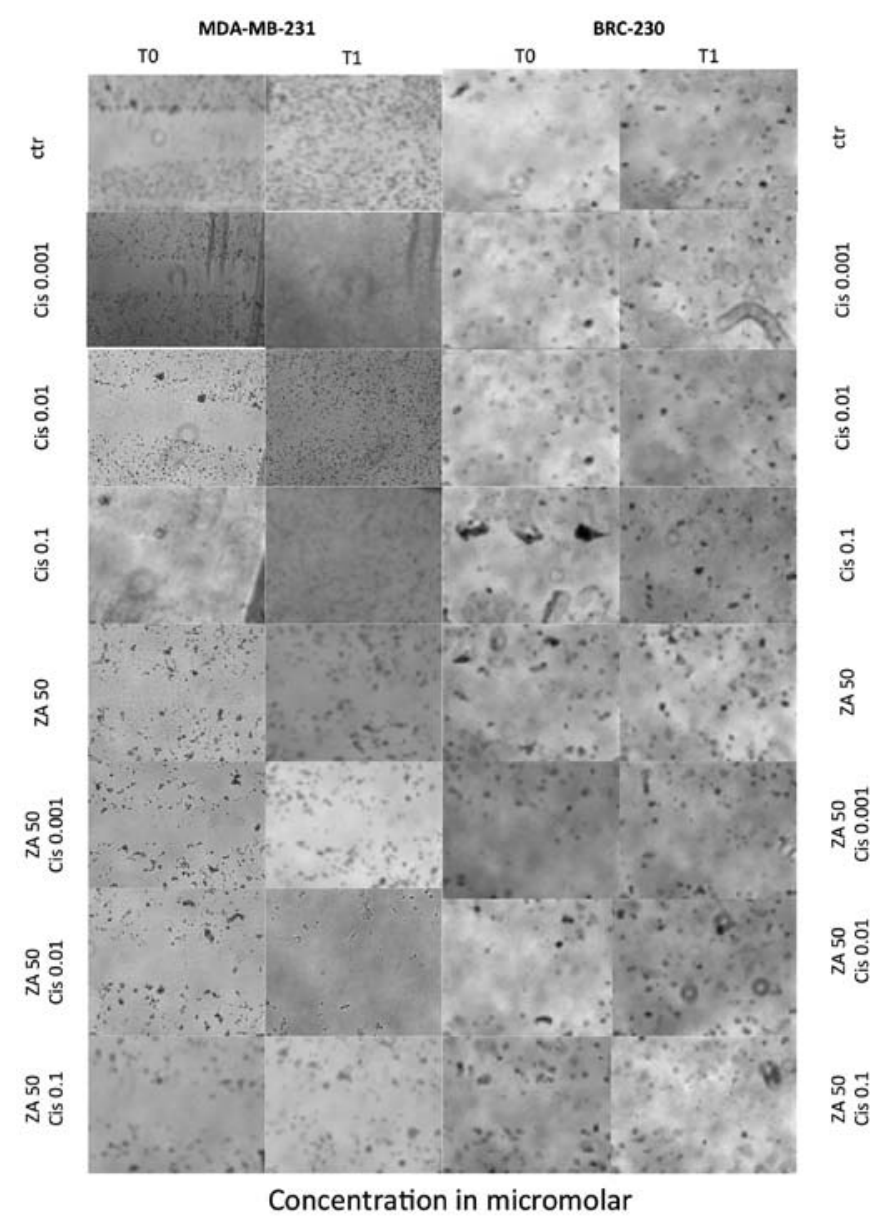

Figure 7. Effect of single and combined treatments on the migration ability detected by wound healing assay. and cells exposed to Cis alone closed the scratch wound by migration, whereas cells treated with ZA alone or in combination with any of the Cis concentrations did not migrate properly and were unable to close the wound (Fig. 7).

\section{Discussion}

In the present study, we evaluated the in vitro effects of different doses of $\mathrm{Cis}$ and ZA, alone or in combination, in breast cancer cell lines. ZA was found to have a direct antitumor activity on breast cancer cells, which is in agreement with results from previous studies $(12,16,18)$. Drug concentrations and exposure times used in our study were chosen on the basis of both literature data (32-34) and results from a preclinical investigation carried out in our laboratory (35), which highlighted that ZA is more effective in triple-negative lines and that a cytocidal effect is reached only in these cells.

We chose platinum compounds to evaluate the potential synergic effect of ZA and chemotherapeutic agents as conventional chemotherapy for breast cancer often uses DNA-damaging drugs to prevent proliferation and stimulate apoptosis of cancer cells, especially in TNBC (36). Initially we performed parallel experiments with carboplatin $(1,10$ and $100 \mu \mathrm{M})$ and $\mathrm{Cis}(0.1,1$ and $10 \mu \mathrm{M})$. As the combination of ZA and carboplatin did not produce additive or synergic effects, we decided to focus on the Cis combination. In the triple-negative cell lines, Cis produced a synergistic effect with ZA in MDA-MB-231, whereas an additive effect was reached in BRC-230. No activity was observed in the other two lines, possibly due to their low sensitivity to these drugs. This finding confirms the results of our previous study which 
highlighted the greater sensitivity of triple-negative cells to ZA. Such sensitivity can be attributed to genetic alterations of oncogenic pathways. K-Ras and BRAF are mutated in the MDA-MB-231 cell line and consequently the K-Ras pathway is constitutively active. BRC-230 has a genetic amplification of EGFR that leads to an overexpression of the protein. We hypothesized that these triple-negative cells are more sensitive to ZA because this drug, inhibiting the mevalonate pathway, may produce a block in the K-Ras pathway which is overactivated in these cells. The hormone receptor-positive (MCF-7) and HER2-expressing (SKBR3) lines, not presenting alterations in BRAF, K-Ras or EGFR genes, are less sensitive. Furthermore, we observed that the two triple-negative lines showed different sensitivity to ZA and Cis, in agreement with a previous study characterizing a cohort of triple-negative breast cancer subtypes with different drug sensitivity (37). Lehmann et al (37) identified 6 subtypes distinguishable by their molecular profiles. The MDA-MB-231 cell line is a mesenchymal stem-like subtype enriched in genes involved in EMT transition and growth pathways, resistant to Cis and sensitive to NVP-BEZ235 and dasatinib. Notably, our results suggest that ZA sensitizes MDA-MB-231 to Cis, in contrast to Cis alone, which did not exert any effect on cell proliferation or survival. We do not have any information on the BRC-230 subtype as this cell line was isolated in our laboratory. Further molecular characterizations are ongoing.

To our knowledge, this is the first study to describe a synergistic effect of ZA in association with Cis in breast cancer cell lines, whereas the combination of these two drugs has already been studied in osteosarcoma (38) and lung cancer cells (39).

We also observed a high inhibition of cell proliferation in MDA-MB-231 when exposed to ZA in association with low concentrations of Cis. Based on these results, we further evaluated two lower concentrations $(0.001$ and $0.01 \mu \mathrm{M})$ of Cis, both of which produced a greater synergistic effect. Finally, we investigated the molecular mechanisms involved in the synergistic/additive effects observed. Assessment of apoptosis showed that the combination of $\mathrm{ZA}$ and $\mathrm{C}$ is induced a small, not statistically significant increase in the percentage of apoptotic cells in both cell lines. Furthermore, we performed chemosensitivity analyses on a primary culture from a bone metastasis specimen as well and the obtained results were concordant with data of a cell line, MCF-7, that has the same pattern of HER-2 and hormonal receptor status of the primary tumor of the bone metastasis specimen. This is a key finding for the confirmation of our in vitro experiments. Further evaluation on a primary culture obtained from a triple negative bone lesion is warranted.

The principal molecular mechanism involved appears to be that of proliferation control. Although only a slight increment in the percentage of cells in the G1 phase was detected, an important decrease in p-MAPK, Mcl-1 and p-mTOR expression and an increase in p21 were observed. P-MAPK is part of the mevalonate pathway and our results thus support previous findings that ZA exerts its effect by modulating this pathway (6). In addition to its anti-apoptotic effect, Mcl-1 has been found to be involved in cell cycle and proliferation regulation (40) and has also been reported to be modulated by $\mathrm{ZA}$ in prostate cancer cell lines (35). mTOR is critically involved in the mediation of cell survival and proliferation, and a number of clinical trials have been conducted on everolimus, a new mTOR inhibitor, in metastatic breast cancer (41). Furthermore, the PI3K/Akt/ mTOR pathway is involved in chemotherapeutic drug resistance and response to radiation in breast cancer cells (42). A previous study highlighted that mTOR inhibitors have the potential to overcome drug resistance from topoisomerase II in solid tumors (43) and ZA is capable of enhancing mTOR inhibition in osteosarcoma cells (44). Finally, we know that MDA-MB-231 is a mesenchymal stem-like subtype cell line that is responsive to mTOR inhibitors but resistant to Cis (37). Taking all these facts into consideration, we can hypothesize that mTOR pathway inhibition plays an important role in ZA anticancer activity and in its ability to overcome MDA-MB-231 resistance to Cis. Further research is warranted to identify new molecular targets to use in preclinical and clinical trials, particularly in TNBC where such targeted therapies are lacking.

In conclusion, our results confirm that ZA exerts a direct antitumor activity on human breast cancer cell lines, as previously described in vitro $(12,16,18)$ in mouse models $(45)$ and as reported in postmenopausal women of the Azure clinical trial (46). Furthermore, we observed that ZA produced a synergistic/additive effect on Cis in triple-negative cell lines, whereas no effect was exerted on the hormone receptor-positive or HER2-expressing lines. Investigating the molecular mechanisms involved, it was concluded that control of proliferation pathways is possibly the key to the action of the drug combination. p21, pMAPK and mTOR pathways were found to be regulated, especially at lower doses of Cis. Although further research is required to elucidate the molecular mechanisms in question, several new potential targets have come to light. Finally, it would be interesting to test the study schedules, first on xenograft models and then in a clinical setting, in an attempt to increase the currently limited options available for triple-negative breast cancer patients. In fact, the synergistic effect exerted by the combination could enable Cis dosages to be reduced, thus minimizing side-effects associated with this chemotherapeutic agent.

\section{Acknowledgements}

The authors thank Gráinne Tierney and Ursula Elbling for editing the manuscript.

\section{References}

1. Jemal A, Bray F, Center MM, Ferlay J, Ward E and Forman D: Global cancer statistics. CA Cancer J Clin 61: 69-90, 2011.

2. Coleman RE and Rubens RD: The clinical course of bone metastases from breast cancer. Br J Cancer 55: 61-66, 1987.

3. Saad F, Adachi JD, Brown JP, Canning LA, Gelmon KA, Josse RG and Pritchard KL: Cancer treatment-induced bone loss in breast and prostate cancer. J Clin Oncol 26: 5465-5476, 2008.

4. Coleman RE: Risks and benefits of bisphosphonates. Br J Cancer 98: 1736-1740, 2008.

5. Coleman RE and McCloskey EV: Bisphosphonates in oncology. Bone 49: 71-76, 2011.

6. Amin D, Cornell SA, Gustafson SK, Needle SJ, Ullrich JW Bilder GE and Perrone MH: Bisphosphonates used for the treatment of bone disorders inhibit squalene synthase in cholesterol biosynthesis. J Lipid Res 33: 1657-1663, 1992.

7. Van Beek E, Pieterman E, Cohen L, Lowick C and Papapoulos S: Farnesyl pyrophosphatase synthase is the molecular target of nitrogen-containing bisphosphonates. Biochem Biophys Res Commun 264: 108-111, 1999 . 
8. Coxon FP, Helfrich MH, Van't Hof R, Sebti S, Ralston SH, Hamilton A and Rogers MJ: Protein geranylgeranylation is required for osteoclast formation, function and survival: inhibition by bisphosphonates and GGTI-298. J Bone Miner Res 15: 1467-1476, 2000.

9. Rogers MJ, Gordon S, Benford HL, Coxon FP, Luckman SP, Monkkonen J and Frith JC: Cellular and molecular mechanisms of action of bisphosphonates. Cancer 88: 2961-2978, 2000.

10. Benford HL, McGowan NW, Helfrich MH, Nuttall ME and Rogers MJ: Visualization of bisphosphonate-induced caspase- 3 activity in apoptotic osteoclasts in vitro. Bone 28: 465-473, 2001

11. Jagdev SP, Coleman RE, Shipman CM, Rostami HA and Croucher PI: The bisphosphonate, ZA, induces apoptosis of breast cancer cells: evidence for synergy with paclitaxel. Br J Cancer 84: 1126-1134, 2001.

12. Senaratne SG, Pirianov G, Mansi JL, Arnett T and Colston KW: Bisphosphonates induce apoptosis in human breast cancer cell lines. Br J Cancer 82: 1459-1468, 2000.

13. Derenne S, Amiot M, Barille S, et al: Zoledronate is a potent inhibitor of myeloma cell growth and secretion of IL-6 and MMP-1 by the tumoral environment. J Bone Miner Res 14: 2048-2056, 1999.

14. Lee MV, Fong EM, Singer FR and Guenette RS: Bisphosphonate treatment inhibits the growth of prostate cancer cells. Cancer Res 61: 2602-2608, 2001.

15. Shipman CM, Rogers MJ, Apperley JF, Russell RG and Croucher PI: Bisphosphonates induce apoptosis in human myeloma cell lines: a novel anti-tumour activity. Br J Haematol 98: 665-672, 1997.

16. Van der Pluijm G, Vloedgraven H, van Beek EJ, van der Wee-Pals L, Lowik C and Papapoulos S: Bisphosphonates inhibit the adhesion of breast cancer cells to bone matrices in vitro. J Clin Invest 98: 698-705, 1996.

17. Boissier S, Magnetto S, Frappart L, Cuzin B, Ebetino FH, Delmas PD and Clezardin P: Bisphosphonates inhibit prostate and breast carcinoma cell adhesion to unmineralized and mineralized bone extracellular matrices. Cancer Res 57: 3890-3894, 1997.

18. Boissier S, Ferreras M, Peyruchaud O, et al: Bisphosphonates inhibit breast and prostate carcinoma cell invasion, an early event in the formation of bone metastases. Cancer Res 60: 2949-2954, 2000.

19. Wood J, Bonjean K, Ruetz S, Bellahcene A, Devy L and Foidart JM: Novel antiangiogenic effects of the bisphosphonate compound zoledronic acid. J Pharmacol Exp Ther 302: 1055-1061, 2002.

20. Neville-Webbe HL, Rostami-Hodjegan A, Evans CA, Coleman RE and Holen I: Sequence- and schedule-dependent enhancement of ZA induced apoptosis by doxorubicin in breast and prostate cancer cells. Int J Cancer 113: 364-371, 2005.

21. Neville-Webbe HL, Evans CA, Coleman RE and Holen I: Mechanisms of the synergistic interaction between the bisphosphonate ZA and the chemotherapy agent paclitaxel in breast cancer cells in vitro. Tumour Biol 27: 92-103, 2006.

22. Vogt U, Bielawski KP, Bosse U and Schlotter CM: Breast tumour growth inhibition in vitro through the combination of cyclophosphamide/metotrexate/5-fluorouracil, epirubicin/cyclophosphamide, epirubicin/paclitaxel, and epirubicin/docetaxel with the bisphosphonates ibandronate and zoledronic acid. Oncol Rep 12: 1109-1114, 2004.

23. Zhao X, Xu X, Guo L, et al: Biomarker alterations with metronomic use of low-dose ZA for breast cancer patients with bone metastases and potential clinical significance. Breast Cancer Res Treat 124: 733-743, 2010.

24. Valentin MD, da Silva SD, Privat M, Alaoui-Jamali M and Bignon YJ: Molecular insights on basal-like breast cancer. Breast Cancer Res Treat 134: 21-30, 2012.

25. Sirohi B, Arnedos M,Popat S, et al: Platinum-based chemotherapy in triple-negative breast cancer. Ann Oncol 19: 1847-1852, 2008

26. Amadori D, Bertoni L, Flamigni A, et al: Establishment and characterization of a new cell line from primary human breast carcinoma. Breast Cancer Res Treat 28: 251-260, 1993.
27. Skehan P, Storeng R, Scudiero D, et al: New colorimetric cytotoxicity assay for anticancer-drug screening. J Natl Cancer Inst 82: 1107-1112, 1990.

28. Monks A, Scudiero D, Skehan P, et al: Feasibility of a high-flux anticancer drug screen using a diverse panel of cultured human tumor cell lines. J Natl Cancer Inst 83: 757-766, 1991.

29. Kern DH, Morgan CR and Hildebrand-Zanki SU: In vitro pharmacodynamics of 1-beta-D-arabinofuranosylcytosine: synergy of antitumor activity with cis-diamminedichloroplatinum(II) Cancer Res 48: 117-121, 1988.

30. Romanelli S, Perego P, Pratesi G, Carenini N, Tortoreto M and Zunino F: In vitro and in vivo interaction between cisplatin and topotecan in ovarian carcinoma systems. Cancer Chemother Pharmacol 41: 385-390, 1998

31. Hahnel A, Wichmann H, Kappler M, Kotzsch M, Vordermark D, Taubert $\mathrm{H}$ and Bache $\mathrm{M}$ : Effects of osteopontin inhibition on radiosensitivity of MDA-MB-231 breast cancer cells. Radiat Oncol 5: 82, 2010

32. Senaratne SG, Mansi JL and Colston KW: The bisphosphonate ZA impairs Ras membrane [correction of impairs membrane] localisation and induces cytochrome c release in breast cancer cells. Br J Cancer 86: 1479-1486, 2002.

33. Rachner TD, Singh SK, Schoppet M, et al: ZA induces apoptosis and changes the TRAIL/OPG ratio in breast cancer cells. Cancer Lett 287: 109-116, 2010.

34. Guise TA: Antitumor effects of bisphosphonates: promising preclinical evidence. Cancer Treat Rev 34: 19-24, 2008.

35. Fabbri F, Brigliadori G, Carloni S, et al: Zoledronic acid increases docetaxel cytotoxicity through pMEK and Mcl-1 inhibition in a hormone-sensitive prostate carcinoma cell line. J Transl Med 6: 43, 2008.

36. Curigliano $\mathrm{G}$ and Goldhirsch A: Triple-negative subtype: new ideas for the poorest prognosis breast cancer. J Natl Cancer Inst Monogr, pp108-110, 2011.

37. Lehmann BD, Bauer JA, Chen X, Sanders ME, Chakravarthy AB, Shyr Y and Pietenpol JA: Identification of human triple-negative breast cancer subtypes and preclinical models for selection of targeted therapies. J Clin Invest 121: 2750-2767, 2011.

38. Benassi MS, Chiechi A, Ponticelli F, et al: Growth inhibition and sensitization to cisplatin by zoledronic acid in osteosarcoma cells. Cancer Lett 250: 194-205, 2007.

39. Ozturk OH, Bozcuk H, Burgucu D, Ekinci D, Ozdogan M, Akca S and Yildiz M: Cisplatin cytotoxicity is enhanced with zoledronic acid in A549 lung cancer cell line: preliminary results of an in vitro study. Cell Biol Int 31: 1069-1071, 2007.

40. Fujise K, Zhang D, Liu J and Yeh ET: Regulation of apoptosis and cell cycle progression by MCL1. Differential role of proliferating cell nuclear antigen. J Biol Chem 275: 39458-39465, 2000.

41. Ellard SL, Clemons M, Gelmon KA, et al: Randomized phase II study comparing two schedules of everolimus in patients with recurrent/metastatic breast cancer: NCI Clinical Trials Group IND.163. J Clin Oncol 27: 4536-4541, 2009.

42. Steelman LS, Navolanic P, Chappell WH, et al: Involvement of Akt and mTOR in chemotherapeutic- and hormonal-based drug resistance and response to radiation in breast cancer cells. Cell Cycle 10: 3003-3015, 2011.

43. Gaur S, Chen L, Yang L, Wu X, Un F and Yen Y: Inhibitors of mTOR overcome drug resistance from topoisomerase II inhibitors in solid tumors. Cancer Lett 311: 20-28, 2011.

44. Moriceau G, Ory B, Mitrofan L, et al: Zoledronic acid potentiates mTOR inhibition and abolishes the resistance of osteosarcoma cells to RAD001 (Everolimus): pivotal role of the prenylation process. Cancer Res 70: 10329-10339, 2010.

45. Ottewell PD, Deux B, Mönkkönen H, Cross S, Coleman RE Clezardin P and Holen I: Differential effect of doxorubicin and zoledronic acid on intraosseous versus extraosseous breast tumor growth in vivo. Clin Cancer Res14: 4658-4666, 2008.

46. Coleman RE, Winter MC, Cameron D, et al: The effects of adding zoledronic acid to neoadjuvant chemotherapy on tumour response: exploratory evidence for direct anti-tumour activity in breast cancer. Br J Cancer 102: 1099-1105, 2010. 